application method. We calculated the exposure intensity level based on application method and use of personal protective equipment. ORs and 95\% CIs were estimated by unconditional logistic regression analyses and adjusted for several potential confounders.

Results 293 case and 3198 control subjects were interviewed. We did not identify positive associations with activities in farming or forestry, pesticide application or pesticide mixing. No consistent positive associations were seen with exposure intensity level scores either. The only statistically significantly raised association in this study was for exposure to chemical fertilisers in forestry (OR 8.93; $95 \%$ CI 1.73 to 42.13 ), but this observation was based on only six exposed subjects. Results did not change when we restricted analyses to morphologically verified cases and excluded proxy interviews as well as cancer controls. We did not observe effect modification by sex or eye colour.

Conclusion Risk estimates for pesticide exposures and occupational activities in agriculture and forestry were not increased and did not indicate a hormonal mechanism due to these exposures. The possible risk increase associated with exposure to chemical fertilisers should be reinvestigated in future studies.

\section{SP1-8 ANAEMIA IN PREGNANT WOMEN ASSISTED BY PUBLIC HEALTHCARE SERVICES OF THE FIVE BRAZILIAN REGIONS BEFORE AND AFTER THE POLICY OF FORTIFICATION OF FLOURS WITH IRON}

doi:10.1136/jech.2011.142976m.85

${ }^{1}$ A P S Sato, ${ }^{1}$ E Fujimori, ${ }^{1}$ S C Szarfarc, ${ }^{1}$ C R M A Araújo, ${ }^{2}$ V A de Oliveira Queiroz, ${ }^{3} \mathrm{R} S \mathrm{~S}$ Moreira-Araújo, ${ }^{4} \mathrm{~S}$ E Priore, ${ }^{5} \mathrm{G} V$ Veiga, ${ }^{6} \mathrm{~T} T$ Uchimura, ${ }^{7} \mathrm{~L} K \mathrm{~K} O$ Yuyama, ${ }^{8} \mathrm{G} S$ Brunken. ${ }^{1}$ University of São Paulo, São Paulo, Brazil; ${ }^{2}$ Federal University of Bahia, Salvador, Bahia, Brazil;, ${ }^{3}$ Federal University of Piauí, Teresina, Piauí, Brazil;, ${ }^{4}$ Federal University of Viçosa, Viçosa, Minas Gerais, Brazil; ${ }^{5}$ Federal University of Rio de Janeiro, Rio de Janeiro, Brazil; ${ }^{6}$ Maringá State University, Maringá, Paraná, Brazil; ${ }^{7}$ National Institute of Amazonian Research, Manaus, Amazonas, Brazil; ${ }^{8}$ Federal University of Mato Grosso, Cuiabá, Mato Grosso, Brazil

Objective To compare prevalence of anaemia and haemoglobin $(\mathrm{Hb})$ levels in pregnant women in the five Brazilian regions, before and after the fortification of flours with iron.

Methods A repeated cross-sectional panel study was carried out by public healthcare services located in the five Brazilian regions. Retrospective data were collected from medical records. Pregnant women were divided into two groups: before-fortification (delivery before June 2004) and after-fortification (last menstrual period after June 2005). The sample included 12119 records. Anaemia was defined as $\mathrm{Hb}<11 \mathrm{~g} / \mathrm{dl}$. We used $\chi^{2}$, Student t test and logistic regression, with significance level of $5 \%$.

Results Anaemia was lower after-fortification of flour $(p<0.05)$. The prevalence dropped from $25 \%$ to $20 \%$ after-fortification $(p<0.001)$. Hb level also was significantly higher after-fortification $(p<0.001)$. The findings showed different sized reductions between regions. In the Northeast and North, where the prevalence of anaemia were high, a significant drop after-fortification was found: from $37 \%$ to $29 \%$, and $32 \%$ to $25 \%$, respectively. In the Southeast and South, whose prevalence was low before-fortification, there were smaller decreases: from $18 \%$ to $15 \%$, and $7 \%$ to $6 \%$, respectively. Logistic regression showed that group, geographic region, marital status, gestational trimester, initial nutritional status and previous pregnancy were associated with anaemia $(\mathrm{p}<0.05)$.

Conclusions The prevalence of anaemia in pregnant women is still high in the Northeast, North and Midwest of Brazil. However, the decrease in anaemia prevalence and increase in $\mathrm{Hb}$ levels suggest a positive effect of fortification of flour to control of iron deficiency. In should be noted that a number of other variables not studied may have contributed to this result.

\section{SP1-9 PREDICTORS OF UNINTENTIONAL POISONING AMONG CHILDREN UNDER-5 YEARS OF AGE IN KARACHI: A MATCHED CASE-CONTROL STUDY}

doi:10.1136/jech.2011.142976m.86

B Ahmed, ${ }^{*}$ Z Fatmi, R Siddiqui. Aga Khan University, Karachi, Pakistan

Introduction Poisoning is the 4th leading cause of unintentional injury in children aged under- 5 years. The study objective was to determine the factors associated with unintentional poisoning among children under- 5 years of age, reporting to emergency rooms (ERs) of tertiary care hospitals in Karachi, Pakistan.

Methods A matched case-control study was conducted on 120 cases and 360 controls. Children with unintentional poisoning were included in the study as cases. For each case three control children matched for age and gender with complaints and diagnosis other than poisoning were selected from the same hospitals ER within $48 \mathrm{~h}$ of case identification. Parents were interviewed using structured questionnaires containing information on socio-demographic factors, child's behaviour, and storage practices. Conditional logistic regression was performed to analyse the data.

Results Accessibility to hazardous chemicals and medicines due to unsafe storage (OR 5.6, 95\% CI 1.9 to 16.7), child's behaviour reported as hyperactive (OR $8.2,95 \%$ CI 4.6 to 16.1), storage of kerosene oil and petrol in soft drink bottles (OR 3.8, 95\% CI 2.0 to 7.3), low socio-economic status (OR 9.2, 95\% CI 2.8 to 30.1), low mothers educational status (OR 4.2, 95\% CI 1.8 to 9.6) and history of previous poisoning (OR 8.6, 95\% CI 1.7 to 43.5) were all independently related to unintentional poisoning.

Conclusion The factors associated with unintentional poisoning in young children are modifiable Key health messages on the safe storage of chemicals and medicines and the use of child resistant containers may help in decreasing the burden of childhood poisoning.

\section{SP1-10 ALCOHOL CONSUMPTION AND SMOKING OF MONGOLIAN ADULTS}

doi:10.1136/jech.2011.142976m.87

${ }^{1} \mathrm{~T}$ Enkh-Oyun, ${ }^{2} \mathrm{D}$ Davaalkham, ${ }^{1} \mathrm{I}$ Chihara, ${ }^{1} \mathrm{R}$ Uehara, ${ }^{1} \mathrm{~K}$ Kotani, ${ }^{1} \mathrm{~A}$ Sadakane, ${ }^{1} \mathrm{Y}$ Aoyama, ${ }^{1} \mathrm{~S}$ Tsuboi, ${ }^{1} \mathrm{R}$ Ae, ${ }^{1} \mathrm{H}$ Takamura, ${ }^{1} Y \mathrm{Y}$ Nakamura. ${ }^{1} \mathrm{Jichi}$ Medical University, Shimotsuke, Tochigi, Japan; ${ }^{2}$ Health Sciences University of Mongolia, Ulaanbaatar, Mongolia

Introduction Alcohol consumption and smoking have being common health problems globally including Mongolia. The objective of this survey was to examine the epidemiology of alcohol consumption and cigarette smoking in the elder population of Mongolia.

Methods A cross-sectional study was carried covering urban and rural areas in Mongolia and used a standardised questionnaire according to WHO STEPwise approach to surveillance manual.

Results A total of 2280 people completed the study. The prevalence of smoking was $24.0 \%$ with significantly more in males $(50.5 \%)$ as compared to in females $(8.3 \%)(p=0.0001)$. Among smokers, mean age of initiation to smoking was 23.5 ( \pm 9.7 ) years and this was differed by sex whereas males started smoking at the age of 21.1 $( \pm 7.8)$ years and females started smoking at the age of 30.2 $( \pm 11.0)$ years. Mean number of cigarettes smoked per day was 11.1 ( \pm 7.8$)$ among current daily smokers. Men smoked $12.3( \pm 8.1)$ cigarettes daily and women smoked $8.1( \pm 6.3)$ cigarettes daily. About six out of ten people using alcohol in last 12 months $(60.3 \%)$. Alcohol consumption was different in gender (in men $75.6 \%$, women $51.2 \%$ ), in age group (in age $40-44$ was $67.0 \%$, in age above 60 was $45.1 \%$ ) and in education level (in primary education $27.7 \%$, in master degree $74.5 \%$ ). Binge drinking was $19.6 \%$ in participants and different by sex (in men $34.2 \%$, in women $10.8 \%, p=0.0001$ ) and location (in rural $24.1 \%$, urban $16.2 \%$ ). 
Conclusion Alcohol consumption and cigarette smoking were different by gender, residence and education level in Mongolian adults.

\section{SP1-11 SELF-REPORTED HEALTH AS A PREDICTOR OF MORTALITY IN ELDERLY MEN LIVING IN SOUTHEAST BRAZIL}

doi:10.1136/jech.2011.142976m.88

L M Santiago, ${ }^{*}$ I E Mattos, L L Luz. Oswaldo Cruz Foundation, National School of Public Health, Rio de Janeiro, Brazil

Introduction Self-reported health is a perception based on an individual interpretation of physical aspects, mental status and expectations and is considered a good predictor of mortality among old people in developed countries. The aim of this study was to evaluate the role of self-reported health as a predictor of mortality in elderly men in a developing country.

Methods The study population consisted of 2875 elderly men of a medium size city in Southeast Brazil, who were followed for 4 years or until the date of their death, whichever occurred first. Individuals alive at the end of follow-up were censored. Multivariate analysis was performed through Cox regression models. Variables presenting statistically significant associations with mortality in bivariate analysis where entered into the models.

Results During the follow-up, 298 deaths occurred. Elders with selfreported poor health presented a greater risk of death, compared to those with self-reported good/excellent health in almost all stratus of the analysed variables. In the final model, poor self-reported health (HR 1.54 95\% CI 1.21 to 1.96), age (HR 1.07 95\% CI 1.06 to 1.09), marital status (HR $1.3295 \%$ CI 1.04 to 1.69), current use of cigarette smoking (HR $1.9495 \%$ CI 1.24 to 2.62), cardiovascular disease (HR 1.62 95\% CI 1.06 to 2.47), diabetes (HR $1.5395 \%$ CI 1.14 to 2.04 ) and recent hospitalisation (HR $1.5095 \%$ CI 1.15 to 1.95) were independently associated to mortality.

Conclusion Self-reported health was a good predictor of mortality in this population of elderly men, even when adjusted for other independent variables. It is important that healthcare services incorporate this indicator in the health evaluation of old people.

\section{SP1-12 RELATIONSHIP BETWEEN SLEEP DURATION AT 3 YEARS OF AGE AND GROWTH DURING CHILDHOOD: A MULTI-LEVEL ANALYSIS}

doi:10.1136/jech.2011.142976m.89

\begin{abstract}
${ }^{1} \mathrm{~K}$ Suzuki, ${ }^{* 2} \mathrm{~A}$ Takahashi, ${ }^{2} \mathrm{M}$ Sato, ${ }^{2} \mathrm{~N}$ Kondo, ${ }^{2} \mathrm{~T}$ Tanaka, ${ }^{2} \mathrm{~A}$ Nagai, ${ }^{1,2} \mathrm{Z}$ Yamagata. ${ }^{1}$ Center for Birth Cohort Studies, Interdisciplinary Graduate School of Medicine and Engineering, University of Yamanashi, Chuo, Yamanashi, Japan; ${ }^{2}$ Department of Health Sciences, Interdisciplinary Graduate School of Medicine and Engineering, University of Yamanashi, Chuo, Yamanashi, Japan
\end{abstract}

Introduction Short sleep duration in early life has been thought to be a risk factor for subsequent overweight. This study aimed to examine the relationship between sleep duration at 3 years of age and childhood weight status through a multi-level analysis.

Methods The study population comprised children born between 1 April 1991, and 31 March 2003, in Koshu City, Japan, and who participated in a medical check-up at 3 years of age. Short and long sleep durations at 3 years of age were the exposures studied. We compared the trajectory of body mass index (BMI) z-scores from 3 to 9 years of age in exposed and non-exposed participants. Random intercepts and slopes model (SAS Proc Mixed) was used for statistical analysis.
Results Of 1794 children who participated in a medical check-up at 3 years of age, 1640 (91.4\%) were not over-weight at 3 years and were followed-up until they were 9 or 10 years old. The number of children in each category of sleep duration, that is, $\leq 9 \mathrm{~h}, 9-10 \mathrm{~h}$, $10-11 \mathrm{~h}$, and $\geq 11 \mathrm{~h}$, was $66(3.7 \%), 609(34.0 \%), 847(47.2 \%)$, and $271(15.1 \%)$, respectively. BMI $z$-scores increased with increase in age $(p=0.03)$ for boys with a short sleep duration $(<9 h)$. On the other hand, sleep duration was not significantly associated with BMI z-score in girls.

Conclusion It was suggested that there is gender difference of the effect of childhood sleep duration on subsequent overweight.

\section{SP1-13 BEING BREASTFED IN INFANCY AND THE RISK OF BREAST CANCER AMONG JAPANESE WOMEN}

doi:10.1136/jech.2011.142976m.90

${ }^{1} \mathrm{Y}$ Minami, ${ }^{*}{ }^{2} \mathrm{Y}$ Nishino, ${ }^{3} \mathrm{M}$ Kawai, ${ }^{4} \mathrm{Y}$ Kakugawa. ${ }^{1}$ Division of Community Health, Tohoku University Graduate School of Medicine, Sendai, Japan; ${ }^{2}$ Division of Epidemiology, Miyagi Cancer Center Research Institute, Natori, Japan; ${ }^{3}$ Department of Surgical Oncology, Tohoku University Graduate School of Medicine, Sendai, Japan; ${ }^{4}$ Division of Surgery, Miyagi Cancer Center Hospital, Natori, Japan

Introduction The incidence of breast cancer is continuously increasing in Japan. The early life exposures such as being breastfed in infancy have been hypothesised to influence subsequent breast cancer risk

Methods We investigated the relationship between having been breastfed and breast cancer risk in a hospital-based case-control study of women aged 30 and over (573 breast cancer cases and 2155 cancer-free controls). Data on reproductive factors, lifestyle, and history of having been breastfed were collected using a selfadministered questionnaire.

Results and Conclusion After adjustment for known risk factors of breast cancer, no association for having been breastfed was observed overall (OR 1.20; 95\% CI 0.82 to 1.75). Analysis stratified according to birth year $(<1950, \geq 1950)$ demonstrated heterogeneity in the association of having been breastfed with breast cancer risk between the two birth-year groups ( $p$ for interaction $=0.0006$ ); having been breastfed was associated with a decreased risk among women who were born before 1950 (OR 0.59, 95\% CI 0.35 to 0.99), whereas it was associated with an increased risk among women born after 1950 (OR 1.65, 95\% CI 0.91 to 2.98). These findings indicate that early nutrition has some effect on breast cancer risk. In Japan, the use of standard formula supplement began to spread around 1950. Endocrine disrupters such as organochlorines were also introduced to the food chain at around the same time. The heterogeneity of breast cancer risk between the two birth-year groups may therefore be attributable to these environmental changes related to infant feeding.

\section{SP1-14 INFLUENCE OF OBESITY INDICATORS ON HYPERTENSION IN ADULTS OF RURAL INDIA}

doi:10.1136/jech.2011.142976m.91

S Anwar, ${ }^{*}$ P Deshmukh, B S Garg. Mahatma Gandhi Institute of Medical Sciences, Sevagram, Wardha, Maharashtra, India

Introduction Being overweight is associated with twofold to sixfold increase in risk of developing hypertension. Studies in urban Indian population also show this association. Present study examines relationship of different anthropometric indicators and blood pressure levels in rural population in central India about which little is known. 